RUHUNA JOURNAL OF SCIENCE

Vol 11 (2): 143-156, December 2020

eISSN: $2536-8400$

http://doi.org/10.4038/rjs.v11i2.93

\title{
Survival modelling of teenage childbirth among Nigerian women
}

O. M. Oladuti

Department of Statistics, The Federal University of Technology, Akure, Nigeria

Correspondence: omoladuti@futa.edu.ng; (D) ORCID: https://orcid.org/0000-0001-9852-2066

Received: $4^{\text {th }}$ April, 2019, Revised: $2^{\text {nd }}$ May, 2020, Accepted: $30^{\text {th }}$ December, 2020

\begin{abstract}
Childbearing is an essential event in woman's life when she neglects her education and career for motherhood. The main cause of population growth in Nigeria is teenage childbearing as reported by many researchers with no significant intervention to reduce this menace. This work is designed to compare the results of Cox, Gompertz and Weibull models with a view to determine the model that best fits the data. To evaluate the effect of some risk factors on the hazard of teenage childbirth among Nigerian women, data obtained from National Demographic Health Survey 2013 was analysed. Results of the analysis showed that having a child at teen age depends on geopolitical zone, location, educational level, circumcision, household wealth index, religion belief, use of contraceptive, whether had a terminated pregnancy, forced sexual acts, awareness of HIV/AIDS, STI and the age of first sexual acts. Akaike Information Criterion (AIC) was employed to evaluate performances of the three models. Weibull regression model has the minimum AIC value compared to both Gompertz and Cox regression models. This shows that Weibull regression model provides best fit to the data.
\end{abstract}

Keywords: Cox proportional hazard model, risk factors, survival time, teenage childbirth

\section{Introduction}

Pubescence is a teenage period between the ages 13 and 19 years which marks the beginning of appearance of sexual characteristics and reproductive maturity. This comprises about twenty percent $(20 \%)$ of the world population with about eighty five percent (85\%) in developing countries (Nwosu 2005). According to Metro (2012), childbirth is the primary killer of teenagers. One of the societal hitches affecting many nations of the world in which Nigeria is not an exception is the childbearing among youngsters. Adolescent childbirth is an issue of enormous concern in societies experiencing high deficiency (Berthoud et al. 2004). In a large scale, one-fifth of 15 
million women under the age of 20 give birth and more than fifty percent of these teenage girls involve in sexual acts before their teen ages (Guttmacher 2004, Maliki 2012). As a result, the rate at which adolescents give birth seems to be on the increase in developing countries (Mahfouz et al. 1995, Adekanle et al. 2008). According to WHO (1991), about 16 million teenagers give birth in developing regions though the prevalence of childbearing among teenagers is declining in the developed nations (Sarantaki and Koutelekos 2002, Hamilton et al. 2007, Hoffman and Maynard 2008). According to United Nation (1997), about one-half of women become mothers before their twentieth birthday. Helen and Anam (2014) examined childbearing trends among 15-19 years old teenagers in Nigeria between 1990 and 2008 in their study. The result showed that the proportions of teenagers who had started childbearing were high but decreased by $6 \%$.

According to Yampolslaaya and Greenbaum (2002), roughly $60 \%$ of mothers who were teenagers lived in poverty at the time of giving birth and their motherhoods were branded with indignity, humiliation, and eventually dropout from schools (Guttmacher 2004, Maliki 2012). Teenage sexual acts in Nigeria have been on increase. The evidences were discovered by the National Demographic Health Survey (NDHS) in 2013, and prevalence around the world and the determinants and its penalties might vary from one region to another. In Sub-Saharan African countries, sexual acts are more prominent and harmful with only $30 \%$ women had their first sexual initiation at the age of 20 and above, 54\% of them have the experience before their $18^{\text {th }}$ birthday, an astounding $24 \%$ indicated that they had not even been 15 yet before experiencing their sexual acts for the first time while over $50 \%$ of young women under age 20 years giving birth to children (Okafor 1997, Aboyeji et al. 2001, Nwosu 2005). This has contributed quite significantly to the fecundity rates observed in the countries of the region, particularly in Nigeria. Nigeria is one of the countries in Africa with high prevalence of teenage pregnancy. Different studies have documented results on teenage pregnancy. Fagbamigbe et al. (2018) analyzed menarche onset timing among Nigerian girls, Cox Proportional Hazard model and Accelerated Failure Time (AFT) models were considered. The result showed that menarche age in Nigeria stretched broadly between age 9 and 22 years and peaked at age 13 and 14 years. Time series analysis techniques ware used by Fagbamigbe et al. (2019) to illustrate variations and trends of teenage pregnancy in Nigeria between 1961 to 2013. The result revealed that one out of every two girls leaving the teen age is likely to have the knowledge of at least one occurrence of pregnancy in Nigeria. The effect of adolescent pregnancy on teenagers in Amassoma community of Southern Ijaw Local Government Area of Bayelsa State in Nigeria was considered by Maliki (2012) using simple percentages. Also, a descriptive crosssectional study of teenage pregnancy and incidence of abortion among teenagers in North Central Nigeria was carried out by Aderibigbe et al. (2011).

Ayo et al. (2016) estimated the regional differences in adolescent childbearing in Nigeria with chi-square test and Cox model. Many of these studies employed 
descriptive statistics and logit model to estimate the impacts of covariates on teenager childbearing which restricted attention to the events that do not occur within the shortest time observed and do not account for the censoring observations. In this study, Cox proportional hazard, Gompertz, and Weibull models were employed to examine the socio-economic and demographic factors influencing adolescent childbirth among Nigerian teenagers and compared them using Akaike Information Criterion (AIC) to determine the most efficient one that fit the data appropriately.

\section{Material and Methods}

\subsection{Source of data and study design}

The data used for this study was obtained from National Demographic and Health Survey (NDHS) conducted in 2013. The design for the collection of data was utilized the fact that there are 37 States including Federal Capital Territory (FCT) in Nigeria. Local Government Areas (LGAs) in each State was subdivided into various localities that were further divided into enumeration areas (EAs). Clusters were used as the primary sampling units based on the enumeration areas. This comprised 372 urban and 532 rural areas across the six geo-political zones in Nigeria.

From the main data of the survey, female respondents aged 15-49 years data with their complete information were extracted on teenager childbirth, i.e., individuals who had their first child at 13-19 years of age. Time to first childbirth of respondents who had their first child between 13-19 years of age inclusively was considered as the survival time in this study and individuals who had not given birth as at the time of survey were right censored. The analyses were based on only 36583 female respondents of age 15-49 years who have the information required for this study.

\subsection{Cox proportional hazard regression model}

Cox Models for survival analysis are usually built from baseline hazard function because there are cases that the exact characteristics of the survivor function are not known but some information is available on how the failure rate changes over time. The survival models measure the failure of an individual at time $t$ and examine the relationship of the survival distribution to covariates under study.

Cox (1972) introduced proportional hazards model which is most frequently used and the simplest member out of a large family of models that focuses directly on the hazard function. Cox proportional hazard model is a semi-parametric, most flexible continuous time model used to investigate covariates effects on hazard function. Cox model makes no assumption about the shape of the baseline hazard function but 
estimates the relationship between the hazard and covariates. For an individual $i$ with covariate vector $x_{i}$, the Cox model is given as

$$
\xi(t / x)=\xi_{o}(t) \exp \left(\beta^{\prime} x\right), t>0
$$

where $\xi(t / x)$ is the hazard of an individual with covariate vector $x, \xi_{o}(t)$ the baseline hazard function, $\beta^{\prime}$ the vector of covariates and $x=\left(x_{1}, x_{2}, \ldots, x_{p}\right)^{\prime}$ the vector of explanatory variables.

The estimated hazard ratio (HR) is simplified as

$$
H R=\frac{\hat{\xi}\left(t, x^{*}\right)}{\hat{\xi}(t, x)}=\frac{\hat{\xi}(t) \exp \left(\sum_{i=1}^{p} \hat{\beta}_{i} x_{i}^{*}\right)}{\hat{\xi}_{o}(t) \exp \left(\sum_{i=1}^{p} \hat{\beta}_{i} x_{i}\right)}=\exp \left\{\sum_{i=1}^{p} \hat{\beta}_{i}\left(x_{i}^{*}-x_{i}\right)\right\}
$$

\subsection{Gompertz Regression Model}

Gompertz is a parametric model in survival analysis. It is a valuable tool in demography and in other scientific disciplines with parameter $\lambda>0$ and $\gamma>0$ which determine the scale and shape of the hazard function respectively.

The survival and hazard functions are given as

$$
s(t)=\exp \left(\frac{\lambda}{\gamma}\left(1-e^{\gamma t}\right)\right) \text { and } h(t)=\lambda \exp (\gamma t), 0 \leq t \leq \infty, \lambda>0
$$

The hazard function of Gompertz proportional hazard model is given as

$$
\xi(t / x)=\lambda e^{\gamma t} \exp \left(\beta^{\prime} x\right), \lambda>0 ; t>0
$$

\subsection{Weibull Regression Model}

The Weibull distribution is another commonly used model for survival analysis that provides estimate of baseline hazard function as well as coefficients for covariates. Weibull distribution is the inclusion of shape parameter to generalization of the exponential distribution which makes it more flexible. Suppose that survival times have a Weibull distribution with scale parameter $\lambda$ and shape parameter $\theta$, the survival and hazard functions are given as

$$
s(t)=e^{-\lambda t^{\theta}} \text { and } h(t)=\theta \lambda t^{\theta-1} ; \lambda>0, \theta>0
$$

Weibull proportional hazard model is given as

$$
\xi(t / x)=\theta \lambda t^{\theta-1} \exp \left(\beta^{\prime} x\right), \lambda>0 ; t>0
$$




\section{Results and Discussion}

From National Demographic and Health Survey 2013, information on whether (or not) the respondents have their first birth at teen ages was extracted, age-at-first birth was recorded in years and those who have their first birth after their teen ages were right censored and those who have never given birth were considered right censored at their current ages. Also, information on categorical covariates which were thought to be associated with response variable were included, namely, Religion (Christianity/ Muslim/ others), Location (urban/ rural area), Level of educational attainment (No education/ Primary/ Secondary/ Higher), Household Wealth Index, HWI (Poorest/ poorer/ Middle/ Richer/ Richest), Current Marital Status (never in union/ Married/ Widowed/ No longer married), Use of Contraceptives (Yes/ No), Ever had a terminated Pregnancy (Yes/ No), Circumcision (Yes/ No), Current age group of the respondents (years 15-19/ 20-24/ 25-29/ 30-39/ 40-49, Ever heard of HIV/AIDS (Yes/ No), Ever heard of STI (Yes/ No), Ever forced to perform unwanted sexual acts (Yes/ No), and Geo-political Zone (North-Central/ North-East/ NorthWest/ South-East/ South-South/ South-West). The continuous covariate Age-at-first sex (in years) was included in the analysis.

\subsection{Descriptive statistics}

Table 1 presents some descriptive information about the 36583 surveyed samples of NDHS data considered. Based on geopolitical zones, the highest number of respondents is from the North-West while South-East has the least number. With respect to locality, 22001 out of 36583 are from rural area. Considering the level of education and age range, the majority was with no education and between 30-39 years. Household wealth index was Richer for the largest number of respondents. Out of 36583, 27343 are yet to be circumcised. With respect to religion, 52.35\% practices Christianity and $46.67 \%$ practices Islam (Muslim). The majority never had a terminated pregnancy nor forced to perform sexual acts. Those who had awareness of HIV/AIDS and Sexual Transmitted Infections (STIs) were the highest number with 17 years as survival time.

\subsection{Models}

The estimated results for model 1 (Table 2), model 2 (Table 3) and model 3 (Table 4) are presented. The estimated hazard ratios with its coefficients for Cox Regression model, Gompertz regression model and Weibull regression model and the P-values with Confidence intervals were used to validate whether each covariate contributed significantly to the model or not. Each factor contribution in the model is determined by its hazard ratio for the three models. 
Table 1: Frequency distribution and survival time of the categorical covariates (total 36583) of surveyed samples.

\begin{tabular}{|c|c|c|c|}
\hline Factors & Factors' Levels & Respondent (\%) & Survival time \\
\hline Geo-Political Zones & $\begin{array}{l}\text { North-West } \\
\text { North-East } \\
\text { North-Central } \\
\text { Southwest } \\
\text { South-East } \\
\text { South-South }\end{array}$ & $\begin{array}{l}8489(23.20) \\
6421(17.55) \\
6075(16.61) \\
5429(14.84) \\
4309(11.78) \\
5860(16.02)\end{array}$ & $\begin{array}{l}18 \\
16 \\
16 \\
16 \\
18 \\
19\end{array}$ \\
\hline Location & $\begin{array}{l}\text { Urban } \\
\text { Rural }\end{array}$ & $\begin{array}{l}14582(39.86) \\
22001(60.14)\end{array}$ & $\begin{array}{l}19 \\
16\end{array}$ \\
\hline Level of Education & $\begin{array}{l}\text { No education } \\
\text { Primary } \\
\text { Secondary } \\
\text { Higher }\end{array}$ & $\begin{array}{r}12542(34.28) \\
6833(18.68) \\
13722(37.51) \\
3486(9.53)\end{array}$ & $\begin{array}{l}16 \\
17 \\
19 \\
19\end{array}$ \\
\hline Circumcision & $\begin{array}{l}\text { Yes } \\
\text { No }\end{array}$ & $\begin{array}{r}9240(25.26) \\
27343(74.74)\end{array}$ & $\begin{array}{l}18 \\
17\end{array}$ \\
\hline Current Age-group & $\begin{array}{l}15-19 \text { years } \\
20-24 \text { years } \\
25-29 \text { years } \\
30-39 \text { years } \\
40-49 \text { years }\end{array}$ & $\begin{array}{l}7384(20.18) \\
6278(17.16) \\
6631(18.13) \\
9495(25.95) \\
6795(18.57)\end{array}$ & $\begin{array}{l}17 \\
17 \\
17 \\
17 \\
16\end{array}$ \\
\hline $\begin{array}{l}\text { Household Wealth Index } \\
\qquad \text { (HWI) }\end{array}$ & $\begin{array}{l}\text { Poorest } \\
\text { Poorer } \\
\text { Middle } \\
\text { Richer } \\
\text { Richest }\end{array}$ & $\begin{array}{l}5961(16.29) \\
7019(19.19) \\
7670(20.97) \\
8057(22.02) \\
7876(21.53)\end{array}$ & $\begin{array}{l}16 \\
16 \\
17 \\
18 \\
18\end{array}$ \\
\hline Religion & $\begin{array}{l}\text { Christianity } \\
\text { Muslim } \\
\text { Others }\end{array}$ & $\begin{array}{c}19153(52.35) \\
17073(46.67) \\
357(0.98)\end{array}$ & $\begin{array}{l}19 \\
16 \\
16\end{array}$ \\
\hline Contraceptive Used & $\begin{array}{l}\text { Yes } \\
\text { No }\end{array}$ & $\begin{array}{r}5930(16.21) \\
30653(83.79)\end{array}$ & $\begin{array}{l}19 \\
17\end{array}$ \\
\hline Ever had a Terminated Pregnancy & $\begin{array}{l}\text { Yes } \\
\text { No }\end{array}$ & $\begin{array}{r}3869(10.58) \\
32714(89.42)\end{array}$ & $\begin{array}{l}17 \\
17\end{array}$ \\
\hline Ever Forced to perform Sexual Acts & $\begin{array}{l}\text { Yes } \\
\text { No }\end{array}$ & $\begin{array}{c}1118(3.06) \\
35465(96.94)\end{array}$ & $\begin{array}{l}18 \\
17\end{array}$ \\
\hline Ever Heard of HIV/AIDS & $\begin{array}{l}\text { Yes } \\
\text { No }\end{array}$ & $\begin{array}{c}33694(92.10) \\
2889(7.90)\end{array}$ & $\begin{array}{l}17 \\
16\end{array}$ \\
\hline Ever Heard of STI & $\begin{array}{l}\text { Yes } \\
\text { No }\end{array}$ & $\begin{array}{c}33946(92.79) \\
2637(7.21)\end{array}$ & $\begin{array}{l}17 \\
16\end{array}$ \\
\hline
\end{tabular}


Table 2: Results of Cox Proportional Hazard model

\begin{tabular}{|c|c|c|c|c|c|c|}
\hline \multicolumn{2}{|l|}{ Covariates } & \multirow{2}{*}{$\begin{array}{l}\text { Hazard } \\
\text { ratio (HR) } \\
1.067\end{array}$} & \multirow{2}{*}{$\begin{array}{c}\text { Coefficient } \\
0.065\end{array}$} & \multirow{2}{*}{$\begin{array}{c}\text { S. E } \\
0.049\end{array}$} & \multirow{2}{*}{$\begin{array}{c}\text { P-value } \\
0.155\end{array}$} & \multirow{2}{*}{$\begin{array}{l}95 \% \text { Conf. } \\
\text { int. of HR } \\
(0.976,1.167)\end{array}$} \\
\hline Geo-political & North-west & & & & & \\
\hline zones & North-East & 1.144 & 0.135 & 0.052 & 0.003 & $(1.046,1.252)$ \\
\hline & North-Central & 1.124 & 0.117 & 0.048 & 0.007 & $(1.033,1.224)$ \\
\hline & Southwest & 0.952 & -0.049 & 0.042 & 0.267 & $(0.873,1.038)$ \\
\hline & South-South & 1.128 & 0.121 & 0.046 & 0.003 & $(1.041,1.223)$ \\
\hline Location & Rural & 0.964 & -0.037 & 0.022 & 0.113 & $(0.921,1.009)$ \\
\hline \multirow{3}{*}{$\begin{array}{l}\text { Level of } \\
\text { education }\end{array}$} & No education & 1.754 & 0.562 & 0.106 & $<0.001$ & $(1.558,1.974)$ \\
\hline & Primary & 1.911 & 0.647 & 0.111 & $<0.001$ & $(1.705,2.141)$ \\
\hline & Secondary & 1.470 & 0.385 & 0.083 & $<0.001$ & $(1.316,1.641)$ \\
\hline Circumcision & & 1.050 & 0.048 & 0.023 & 0.026 & $(1.006,1.095)$ \\
\hline \multirow{4}{*}{$\begin{array}{l}\text { Current marital } \\
\text { status }\end{array}$} & Single & 0.169 & -1.776 & 0.012 & $<0.001$ & $(0.147,0.195)$ \\
\hline & Married & 1.065 & 0.063 & 0.052 & 0.198 & $(0.967,1.173)$ \\
\hline & Widowed & 1.254 & 0.226 & 0.083 & 0.001 & $(1.102,1.427)$ \\
\hline & $15-19$ years & 0.638 & -0.449 & 0.024 & $<0.001$ & $(0.592,0.688)$ \\
\hline \multirow{3}{*}{$\begin{array}{l}\text { Current age- } \\
\text { groups }\end{array}$} & $25-29$ years & 0.860 & -0.151 & 0.023 & $<0.001$ & $(0.817,0.905)$ \\
\hline & 30-39 years & 0.825 & -0.193 & 0.020 & $<0.001$ & $(0.786,0.865)$ \\
\hline & $40-49$ years & 0.810 & -0.211 & 0.022 & $<0.001$ & $(0.769,0.853)$ \\
\hline \multirow{4}{*}{$\begin{array}{l}\text { Household } \\
\text { wealth index }\end{array}$} & Poorer & 1.074 & 0.071 & 0.025 & 0.003 & $(1.025,1.125)$ \\
\hline & Middle & 1.146 & 0.136 & 0.032 & $<0.001$ & $(1.086,1.210)$ \\
\hline & Richer & 1.060 & 0.058 & 0.035 & 0.079 & $(0.993,1.130)$ \\
\hline & Richest & 0.876 & -0.132 & 0.038 & 0.002 & $(0.805,0.953)$ \\
\hline \multirow[t]{2}{*}{ Religion } & Christianity & 0.937 & -0.065 & 0.074 & 0.408 & $(0.803,1.093)$ \\
\hline & Muslim & 0.963 & -0.038 & 0.075 & 0.625 & $(0.827,1.121)$ \\
\hline \multicolumn{2}{|c|}{ Contraceptive used } & 0.886 & -0.121 & 0.024 & $<0.001$ & $(0.840,0.935)$ \\
\hline \multicolumn{2}{|c|}{ Ever had a terminated pregnancy } & 1.066 & 0.064 & 0.027 & 0.010 & $(1.015,1.120)$ \\
\hline \multicolumn{2}{|c|}{ Forced sexual acts } & 1.230 & 0.207 & 0.067 & $<0.001$ & $(1.106,1.368)$ \\
\hline \multicolumn{2}{|c|}{ Ever heard of HIV/AIDS } & 0.884 & -0.124 & 0.077 & 0.155 & $(0.745,1.048)$ \\
\hline \multicolumn{2}{|c|}{ Ever heard of STI } & 1.092 & 0.088 & 0.992 & 0.334 & $(0.914,1.304)$ \\
\hline \multicolumn{2}{|l|}{ Age at first sex } & 0.711 & -0.340 & 0.003 & $<0.001$ & $(0.707,0.716)$ \\
\hline
\end{tabular}


Table 3: Results of Weibull Regression Model

\begin{tabular}{|c|c|c|c|c|c|c|}
\hline \multicolumn{2}{|l|}{ Covariates } & \multirow{2}{*}{$\begin{array}{c}\begin{array}{c}\text { Hazard } \\
\text { ratio }(\mathrm{HR})\end{array} \\
1.238\end{array}$} & \multirow{2}{*}{$\begin{array}{c}\text { Coefficient } \\
0.213\end{array}$} & \multirow{2}{*}{$\begin{array}{c}\text { S. E } \\
0.057\end{array}$} & \multirow{2}{*}{$\begin{array}{c}\text { P-value } \\
<0.001\end{array}$} & \multirow{2}{*}{$\begin{array}{l}\begin{array}{l}95 \% \text { Conf. } \\
\text { int. of HR }\end{array} \\
(1.131,1.355)\end{array}$} \\
\hline Geo-political & North-west & & & & & \\
\hline zones & North-East & 1.189 & 0.173 & 0.055 & $<0.001$ & $(1.087,1.302)$ \\
\hline & North-Central & 1.303 & 0.264 & 0.056 & $<0.001$ & $(1.197,1.418)$ \\
\hline & Southwest & 1.107 & 0.102 & 0.049 & 0.022 & $(1.015,1.207)$ \\
\hline & South-South & 1.243 & 0.218 & 0.051 & $<0.001$ & $(1.147,1.347)$ \\
\hline Location & Rural & 0.967 & -0.034 & 0.023 & 0.145 & $(0.923,1.012)$ \\
\hline \multirow{3}{*}{$\begin{array}{l}\text { Level of } \\
\text { education }\end{array}$} & No education & 2.522 & 0.925 & 0.155 & $<0.001$ & $(2.237,2.845)$ \\
\hline & Primary & 2.998 & 1.098 & 0.176 & $<0.001$ & $(2.672,3.364)$ \\
\hline & Secondary & 2.041 & 0.713 & 0.115 & $<0.001$ & $(1.827,2.280)$ \\
\hline Circumcision & & 1.015 & 0.015 & 0.021 & 0.487 & $(0.973,1.059)$ \\
\hline \multirow{4}{*}{$\begin{array}{l}\text { Current marital } \\
\text { status }\end{array}$} & Single & 0.157 & -1.849 & 0.011 & $<0.001$ & $(0.137,0.181)$ \\
\hline & Married & 1.236 & 0.212 & 0.061 & $<0.001$ & $(1.122,1.362)$ \\
\hline & Widowed & 1.344 & 0.295 & 0.089 & $<0.001$ & $(1.181,1.530)$ \\
\hline & $15-19$ years & 0.636 & -0.453 & 0.024 & $<0.001$ & $(0.590,0.685)$ \\
\hline \multirow{3}{*}{$\begin{array}{l}\text { Current age- } \\
\text { groups }\end{array}$} & $25-29$ years & 0.719 & -0.330 & 0.019 & $<0.001$ & $(0.683,0.757)$ \\
\hline & 30-39 years & 0.569 & -0.563 & 0.014 & $<0.001$ & $(0.542,0.598)$ \\
\hline & $40-49$ years & 0.416 & -0.878 & 0.012 & $<0.001$ & $(0.393,0.439)$ \\
\hline \multirow{4}{*}{$\begin{array}{l}\text { Household } \\
\text { wealth index }\end{array}$} & Poorer & 1.060 & 0.058 & 0.025 & 0.015 & $(1.011,1.110)$ \\
\hline & Middle & 1.157 & 0.146 & 0.032 & $<0.001$ & $(1.096,1.222)$ \\
\hline & Richer & 1.125 & 0.118 & 0.037 & $<0.001$ & $(1.054,1.201)$ \\
\hline & Richest & 0.787 & -0.240 & 0.034 & $<0.001$ & $(0.722,0.857)$ \\
\hline \multirow[t]{2}{*}{ Religion } & Christianity & 0.925 & -0.078 & 0.073 & 0.326 & $(0.792,1.081)$ \\
\hline & Muslim & 0.932 & -0.070 & 0.073 & 0.369 & $(0.800,1.086)$ \\
\hline \multicolumn{2}{|c|}{ Contraceptive used } & 0.764 & -0.269 & 0.021 & $<0.001$ & $(0.724,0.807)$ \\
\hline \multicolumn{2}{|c|}{ Ever had a terminated pregnancy } & 1.044 & 0.044 & 0.026 & 0.082 & $(0.994,1.097)$ \\
\hline \multicolumn{2}{|c|}{ Forced sexual acts } & 1.157 & 0.146 & 0.063 & 0.007 & $(1.041,1.287)$ \\
\hline \multicolumn{2}{|c|}{ Ever heard of hiv/aids } & 0.733 & -0.310 & 0.064 & $<0.001$ & $(0.618,0.869)$ \\
\hline \multicolumn{2}{|l|}{ Ever heard of sti } & 1.305 & 0.266 & 0.118 & 0.003 & $(1.093,1.559)$ \\
\hline \multicolumn{2}{|l|}{ Age at first sex } & 0.734 & -0.309 & 0.002 & $<0.001$ & $(0.729,0.739)$ \\
\hline
\end{tabular}


Table 4: Results of Gompertz Regression model

\begin{tabular}{|c|c|c|c|c|c|c|}
\hline \multicolumn{2}{|l|}{ Covariates } & \multirow{2}{*}{$\begin{array}{c}\begin{array}{c}\text { Hazard } \\
\text { ratio }(\mathrm{HR})\end{array} \\
1.220\end{array}$} & \multirow{2}{*}{$\begin{array}{c}\text { Coefficient } \\
0.199\end{array}$} & \multirow{2}{*}{$\begin{array}{c}\text { S. E } \\
0.056\end{array}$} & \multirow{2}{*}{$\begin{array}{l}\text { P-value } \\
<0.001\end{array}$} & \multirow{2}{*}{$\begin{array}{l}95 \% \text { Conf. int. } \\
\text { of HR } \\
(1.114,1.335)\end{array}$} \\
\hline Geo-political zones & North-west & & & & & \\
\hline & North-East & 1.141 & 0.132 & 0.053 & 0.004 & $(1.043,1.249)$ \\
\hline & North-Central & 1.283 & 0.250 & 0.056 & $<0.001$ & $(1.179,1.397)$ \\
\hline & Southwest & 1.112 & 0.106 & 0.049 & 0.016 & $(1.020,1.213)$ \\
\hline & South-South & 1.247 & 0.221 & 0.051 & $<0.001$ & $(1.151,1.352)$ \\
\hline Location & Rural & 0.974 & -0.026 & 0.023 & 0.262 & $(0.931,1.020)$ \\
\hline \multirow[t]{3}{*}{ Level of education } & No education & 2.401 & 0.876 & 0.147 & $<0.001$ & $(2.129,2.707)$ \\
\hline & Primary & 2.884 & 1.059 & 0.169 & $<0.001$ & $(2.570,3.236)$ \\
\hline & Secondary & 2.034 & 0.710 & 0.115 & $<0.001$ & $(1.820,2.272)$ \\
\hline Circumcision & & 1.010 & 0.010 & 0.022 & 0.631 & $(0.969,1.054)$ \\
\hline \multirow{4}{*}{$\begin{array}{l}\text { Current marital } \\
\text { status }\end{array}$} & Single & 0.159 & -1.836 & 0.011 & $<0.001$ & $(0.138,0.184)$ \\
\hline & Married & 1.248 & 0.222 & 0.062 & $<0.001$ & $(1.133,1.375)$ \\
\hline & Widowed & 1.272 & 0.241 & 0.084 & $<0.001$ & $(1.118,1.448)$ \\
\hline & $15-19$ years & 0.585 & -0.536 & 0.022 & $<0.001$ & $(0.543,0.630)$ \\
\hline \multirow[t]{3}{*}{ Current age-groups } & $25-29$ years & 0.751 & -0.286 & 0.019 & $<0.001$ & $(0.714,0.791)$ \\
\hline & 30-39 years & 0.610 & -0.494 & 0.015 & $<0.001$ & $(0.582,0.641)$ \\
\hline & 40-49 years & 0.402 & -0.912 & 0.012 & $<0.001$ & $(0.380,0.425)$ \\
\hline \multirow{4}{*}{$\begin{array}{l}\text { Household wealth } \\
\text { index }\end{array}$} & Poorer & 1.058 & 0.057 & 0.025 & 0.017 & $(1.010,1.109)$ \\
\hline & Middle & 1.154 & 0.143 & 0.032 & $<0.001$ & $(1.093,1.218)$ \\
\hline & Richer & 1.121 & 0.115 & 0.037 & 0.001 & $(1.051,1.197)$ \\
\hline & Richest & 0.794 & -0.231 & 0.035 & $<0.001$ & $(0.729,0.864)$ \\
\hline \multirow[t]{2}{*}{ Religion } & Christianity & 0.929 & -0.732 & 0.073 & 0.354 & $(0.796,1.085)$ \\
\hline & Muslim & 0.931 & -0.712 & 0.073 & 0.361 & $(0.799,1.085)$ \\
\hline \multicolumn{2}{|l|}{ Contraceptive used } & 0.767 & -0.265 & 0.021 & $<0.001$ & $(0.727,0.810)$ \\
\hline \multicolumn{2}{|c|}{ Ever had a terminated pregnancy } & 1.037 & 0.037 & 0.026 & 0.143 & $(0.988,1.090)$ \\
\hline \multicolumn{2}{|l|}{ Forced sexual acts } & 1.109 & 0.103 & 0.060 & 0.056 & $(0.997,1.233)$ \\
\hline \multicolumn{2}{|c|}{ Ever heard of HIV/AIDS } & 0.733 & -0.311 & 0.064 & $<0.001$ & $(1.118,1.448)$ \\
\hline \multicolumn{2}{|c|}{ Ever heard of STI } & 1.297 & 0.260 & 0.118 & 0.004 & $(1.086,1.550)$ \\
\hline \multicolumn{2}{|l|}{ Age at first sex } & 0.753 & -0.284 & 0.002 & $<0.001$ & $(0.748,0.758)$ \\
\hline
\end{tabular}


Table 5: Summary of the results (hazard ratio) of three models.

\begin{tabular}{|c|c|c|c|c|}
\hline \multicolumn{2}{|l|}{ Covariates } & \multicolumn{3}{|c|}{ Hazard ratio } \\
\hline & & $\operatorname{Cox}$ & Weibull & Gompertz \\
\hline \multirow[t]{5}{*}{ Geo-political zones } & North-west & 1.067 & $1.238 *$ & $1.220^{*}$ \\
\hline & North-East & $1.144 *$ & $1.189 *$ & $1.141 *$ \\
\hline & North-Central & $1.124 *$ & $1.303^{*}$ & $1.283^{*}$ \\
\hline & Southwest & 0.952 & $1.107 *$ & $1.112 *$ \\
\hline & South-South & $1.128 *$ & $1.243 *$ & $1.247 *$ \\
\hline Location & Rural & 0.964 & 0.967 & 0.974 \\
\hline \multirow[t]{3}{*}{ Level of education } & No education & $1.754^{*}$ & $2.522 *$ & $2.401 *$ \\
\hline & Primary & $1.911 *$ & $2.998 *$ & $2.884^{*}$ \\
\hline & Secondary & $1.470^{*}$ & $2.041 *$ & $2.034 *$ \\
\hline Circumcision & & $1.050 *$ & 1.015 & 1.010 \\
\hline \multirow[t]{4}{*}{ Current marital status } & Single & $0.169 *$ & $0.157 *$ & $0.159 *$ \\
\hline & Married & 1.065 & $1.236^{*}$ & $1.248 *$ \\
\hline & Widowed & $1.254^{*}$ & $1.344 *$ & $1.272 *$ \\
\hline & 15-19 years & $0.638 *$ & $0.636^{*}$ & $0.585^{*}$ \\
\hline \multirow[t]{3}{*}{ Current age-groups } & $25-29$ years & $0.860 *$ & $0.719 *$ & $0.751 *$ \\
\hline & $30-39$ years & $0.825^{*}$ & $0.569 *$ & $0.610^{*}$ \\
\hline & $40-49$ years & $0.810^{*}$ & $0.416^{*}$ & $0.402 *$ \\
\hline \multirow{4}{*}{$\begin{array}{l}\text { Household wealth } \\
\text { index }\end{array}$} & Poorer & $1.074 *$ & $1.060 *$ & $1.058 *$ \\
\hline & Middle & $1.146^{*}$ & $1.157 *$ & $1.154 *$ \\
\hline & Richer & 1.060 & $1.125 *$ & $1.121 *$ \\
\hline & Richest & $0.876^{*}$ & $0.787 *$ & $0.794 *$ \\
\hline \multirow[t]{2}{*}{ Religion } & Christianity & 0.937 & 0.925 & 0.929 \\
\hline & Muslim & 0.963 & 0.932 & 0.931 \\
\hline \multicolumn{2}{|l|}{ Contraceptive used } & $0.886^{*}$ & $0.764 *$ & $0.767 *$ \\
\hline \multicolumn{2}{|c|}{ Ever had a terminated pregnancy } & $1.066^{*}$ & 1.044 & 1.037 \\
\hline \multicolumn{2}{|c|}{ Forced sexual acts } & $1.230^{*}$ & $1.157 *$ & $1.109 *$ \\
\hline \multicolumn{2}{|l|}{ Ever heard of hiv/aids } & 0.884 & $0.733 *$ & $0.733^{*}$ \\
\hline \multicolumn{2}{|l|}{ Ever heard of sti } & 1.092 & $1.305 *$ & $1.297 *$ \\
\hline \multicolumn{2}{|l|}{ Age at first sex } & $0.711^{*}$ & $0.734^{*}$ & $0.753^{*}$ \\
\hline
\end{tabular}

*significance $\mathrm{p}<0.05$ 
Table 5 shows the summary of the results comprising the estimated hazard ratio (HR), coefficients, standard errors and confidence intervals for Cox proportional hazard, Weibull Regression and Gompertz Regression models respectively.

Based on geopolitical zones, those who are in North-East, North-West, NorthCentral, South-West and South-South were more likely to experience adolescent childbirth while South-West were less likely with Cox model compare to those who are in South-East. Those who reside in rural area were less likely to have teenage childbirth compared to their counterpart. From the level of education, those that have no education, primary and secondary education were more likely to have teenage childbirth $(\mathrm{P}<0.001)$ compared to those who have higher education. Nevertheless, with Gompertz and Weibull models, respondents with no education, primary and secondary education were significantly $(\mathrm{P}<0.001)$ twice more likely to give birth at teen age than those with higher education. Those that were not circumcised were more likely to have their first child at their teen period than those that were circumcised.

Based on their current marital status, respondents who are presently single were less prone to teenage childbirth $(\mathrm{P}<0.001)$ while those who are currently married and widowed were more likely to have teen childbirth at their teen age compared to those divorced/ separated. Those within age-groups 15-19, 25-29, 30-39 and 40-49 years had the hazard ratios of which are significantly $(\mathrm{P}<0.001)$ lower than those aged 20 24 years which implies that there were less teenage childbirths in those age groups.

Based on the household economic status variable measure by wealth index, those who are poorer, middle and richer were more likely to give birth for the first time at their teen age while the richest were less prone to it compared to the poorest.

According to the results based on the religion belief, the respondents who practice Christianity and Muslim were less likely to have teenage childbirth than others. Respondents that are not using contraceptive were less prone to teenage childbirth compared to those that are using it. Those that had never terminated pregnancy were more likely to have to teenage childbirth than their counterparts. For those who were never forced to perform unwanted sexual acts, they were more likely to have a baby at their teen age compared to their counterparts.

Those who heard of HIV/AIDS were less prone to teenage childbirth compared to those who did not, and also those who heard of STI were more likely to have their first child at teen age compared to their counterparts. The hazard of teenage childbirth decreases with a one-year increase in age at first sex by $0.711(\mathrm{P}<0.001)$ which means the earlier the age at first sexual initiation the greater the chance of having a child at teen age.

Akaike Information Criteria were used to compare the three models under proportional hazards framework of survival data. The relative performances of the models are shown in Table 6. It was observed that Weibull has the least value $(\mathrm{AIC}=22488.98)$ which shows best performance while AIC values of Cox regression and Gompertz models are 283793.1 and 31343.8, respectively. 
Table 6: Comparison of relative performances of Cox, Gompertz and Weibull models.

\begin{tabular}{lccr}
\hline Models & Number of Observations & Log-likelihood & \multicolumn{1}{c}{ AIC } \\
\hline Cox & 36583 & -141867.60 & 283793.00 \\
Weibull & 36583 & -11213.49 & 22488.98 \\
Gompertz & 36583 & -15640.90 & 31343.80 \\
\hline
\end{tabular}

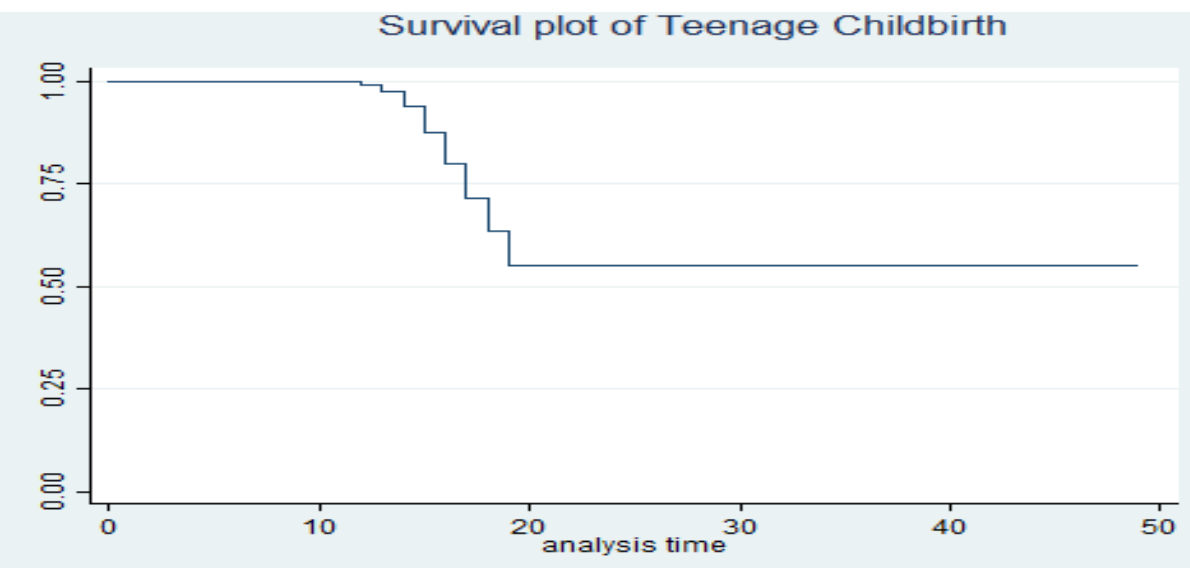

Hazard plot of Teenage Childbirth

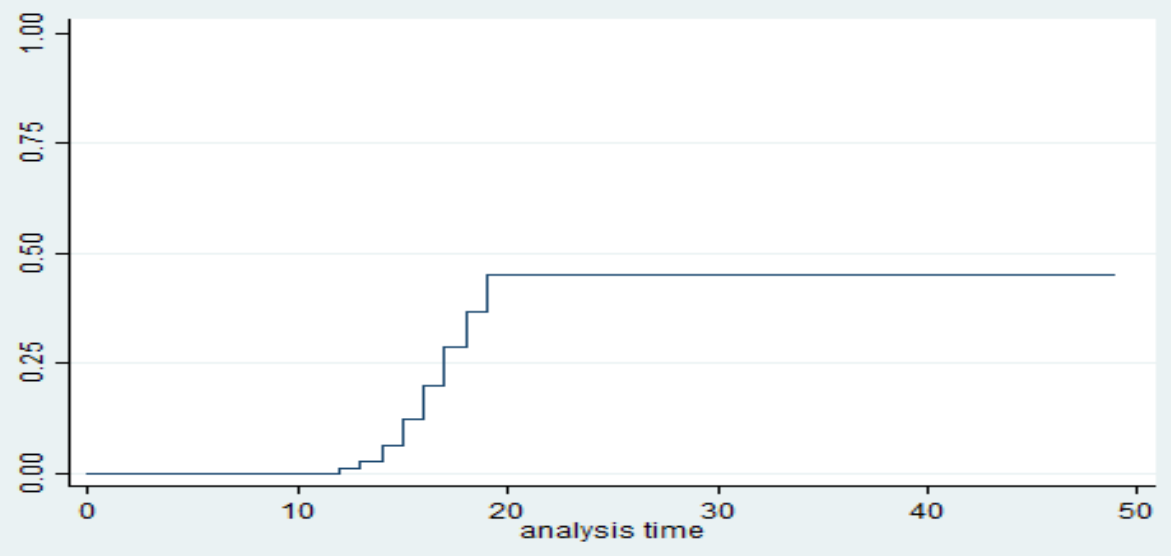

Fig 1. Kaplan-Meier (K-M) plots of Survival and hazard functions of time.

Plots of the Kaplan-Meier (K-M) curves to the survival and hazard experience of time-to-teenage childbirth presented in Figure 1 show that the event occur between ages 16 and 19. 


\section{Conclusions}

We have successfully modelled the prevalence of teenage childbirth and the risk factor associated with it among Nigerian Women. Data used to model the teenage childbearing were obtained from National Demographic Health Survey 2013 and the Cox regression model was applied to test the effect of each covariate (categorical and continuous) in the hazard rate. The results showed the rate of teen age childbearing was related in all geo-political areas though slightly higher with people from NorthEast, North-West, North-Central, South-West and South-South. Those living in urban area had higher risk of given birth at teen age compare to those in rural areas. A respondent with no education, primary and secondary has a tendency of given birth at teen age. The risk of teenage childbirth for those who were not circumcised was slightly higher. Respondents whose household wealth index is richest were less prone to teenage childbirth. Based on their religion belief, those who practice Christianity and Muslim have the lower risk of teenage childbirth than other. Non usage of contraceptives decreases risk of teenage childbirth. Respondents who had never terminated pregnancy nor forced to perform sexual acts have higher risk of teenage childbirth. Those who are aware of sexual transmitted infections (STIs) have higher risk of teenage childbirth than those who heard of HIV/AIDS. We also observed that the higher the age at first sexual acts, the lower the risk of teenage childbirth. It might be because of knowledge acquired based on level of education or awareness through social media on sexual transmitted infections (STIs) and menace of having a child at teen age.

Based on the three models, the results revealed that Weibull regression model is the best since it has the least AIC value. This shows that the parametric Weibull regression model could better determine the factors associated with teenage childbearing than both the parametric Gompertz model and Semi-parametric Cox model, respectively.

\section{Acknowledgments}

Two anonymous reviewers are acknowledged for valuable comments on the initial draft of the manuscript.

\section{References}

Aboyeji AP, Fawole AA, Ijaiya MA. 2001. Knowledge and previous contraceptive use by pregnant teenagers in Ilorin, Nigeria. Tropical Journal of Obstetrics and Gynecology 18(2):73-76.

Aderibigbe S, Araoye M, Akande T, Olumuyiwa MJ, Ibrahim MO, Babatunde O. 2010. Teenage Pregnancy and Prevalence of Abortion among In-school Adolescents in North-Central, Nigeria. Asian social science 7(1): 122-123

Adekanle DA, Adeyemi AS, Odu OO. 2008. Teenage and nonteenage pregnant women in southwestern Nigeria: a descriptive study. Calicut Medical Journal 6 (3): 693, e5 
Adeniyi F, Fagbamigbe Rotimi F, Afolabi Oyindamola, B Yusuf. 2019. Trend analysis of teenage pregnancy in Nigeria (1961-2013): How effective is the contraceptive use campaign? International Journal of Public Health Science 8 (2): 163-173.

Ayo S, Adebowale, Adeniyi F, Fagbamigbe, Ayodeji M Adebayo. 2016. Regional Differences in Adolescent Childbearing in Nigeria. Journal of Population and Social Studies 24(2): 101-116. DOI 10.14456/jpss.2016.

Berthoud R, Ermisch J, Fransesconi M, Liao T, Pevalin D, Robson K. 2004. Long term consequences of teenage births for parents and their children. Second Edition, Jessica Kingsley Publishers, London and Philadelphia.

Cox DR. 1972. Regression Models and Life Tables. Journal of the Royal Statistical Society Series B (Methodological) 34: 187-220.

Fagbamigbe AF, Obiyan MO, Fawole OI. 2018. Parametric Survival analysis of menarche onset timing among Nigerian girls. Heliyon 4 (2018) e01105. doi: 10.1016/j.heliyon.2018. e01105

Guttmacher. 2004. Sex and America's teenagers. Available at www.guttmacher.org. Accessed on March 7, 2011.

Hamilton BE, Martin JA, Ventura SJ. 2007. Births preliminary data for 2006. Hyattsville, MD: National Center for Health Statistics. National Vital Statistics Reports 56(7).

Helen NA, Anam B. 2014. Teenage childbearing trend in Nigerian 1990-2008. Education and Science Journal of Policy Review and Curriculum Development 4(1): 1741- 8771.

Hoffman SD, Maynard RA. 2008. Kids Having Kids: Economic costs and social consequences of teen pregnancy. Second Edition, Urban Institute Press, Washington DC.

Jiezhi Q. 2009. Comparison of proportional hazards and accelerated failure time models. Thesis submited to the College of Graduate Studies and Research, University of Saskatchewan, Department of Mathematics and Statistics.

Maliki AE. 2012. The effect of teenage pregnancy on adolescents in Amassoma Community of Southern Ijaw Local Government Area of Bayelsa State. Asian Social Science 8. 10.5539/ass. v8n1p62.

Mahfouz AA, el-Said MM, al-Erian RA, Hamid AM. 1995. Teenage pregnancy: Are teenagers a highrisk group? European Journal of Obstetrics \& Gynecology and Reproductive Biology 59 (1): 17-20.

Metro. 2012. Childbirth is the number one killer of teenagers. Metro News. Published June 27, 2012.

Nwosu AN. 2005. Effects of health education on the reproductive health behavior of in-school adolescent girls in Enugu. Doctoral dissertation, University of Nigeria.

Okafor A. 1997. Sexual knowledge and sources of sexual information of secondary school students in Anambra State, Nigeria. Health and movement education Journal 1(1): 9-15.

Svetlana Y, Eric CB, Paul EG. 2002. Early pregnancy among adolescent females with serious emotional disturbances risk factors and outcomes. Journal of Emotional and Behavioral Disorders 10 (2):108115 .

Sarantaki A, Koutelekos I. 2002. Teenage pregnancy. Health Science Journal 1 (2): 1-6.

United Nation. 1997. The right to reproductive and sexual health. United Nations Department of public information.

Viegas OA, Wiknsosatro G, Sahagun GH, Chaturachinda K, Ratnam SS. 1992. Safe childbirth needs more than medical services. World Health forum 13: 59-65.

WHO 1991. World Health Organisation, Maternal and mortality ratios and rates: a tabulation of available information. $3^{\text {rd }}$ edition. Geneva Unpublished report.

WHO 2005. World Health Organisation- Facts and figures from the world health report.

WHO 2013. World Health Organization, Nigeria: Maternal and perinatal country profile 13(1), Geneva. http://www.who.int/maternal_child_adolescent/epidemiology/ profiles/maternal/ nga.pdf, Accessed on February 9, 2013.

Yampolskaya S, Brown E, Greenbaum P. 2002. Early pregnancy among adolescent females with serious emotional disturbances: Risk factors and outcomes. Journal of Emotional and Behavioral Disorders 10(2): 108-115. 